\title{
B cells join T cell clusters in the host response to recurrent herpes simplex virus 2 infection
}

\author{
Jeff R. Gehlhausen, ${ }^{1}$ and Akiko Iwasaki ${ }^{1,2,3,4}$ \\ 'Department of Dermatology and 2Department of Immunobiology, Yale University School of Medicine, New Haven, Connecticut, USA. ${ }^{3}$ Department of Molecular Cellular and Developmental Biology, Yale \\ University, New Haven, Connecticut, USA. ${ }^{4}$ Howard Hughes Medical Institute.
}

\begin{abstract}
Recurrent genital herpes lesions are infiltrated by various leukocytes, yet the role of B cell subsets in this process is unknown. In this issue of the $J C l$, Ford et al. describe the presence and antibody-secreting role of local B cell populations in herpes simplex virus 2 (HSV-2) recurrent lesions. The authors analyzed a comprehensive array of sequential skin biopsy specimens from HSV-2-infected patients over time and at various stages of infection. Using immunofluorescence and in situ hybridization, the authors show the presence of rare $\lg \mathrm{D}^{+}$naive $B$ cells and IgG-expressing antibody-secreting cells (ASCs) in recurrent HSV-2 lesions embedded in CD4+ T cell-rich dermal immune infiltrates, levels of which transiently increase during lesion reactivation and healing. Notably, local increases in HSV-2specific antibodies in recurrent lesions were detected, whereas serum HSV-2 antibody levels remained stable. Future research is needed to understand the precise role of these tissue-visiting B cells in disease resolution.
\end{abstract}

\section{HSV-2 and human disease}

Herpes simplex virus 2 (HSV-2) is a major cause of genital ulcers worldwide and among the most common sexually transmitted infections (STIs), with seroprevalence studies demonstrating infection in more than $15 \%$ of the US population (1-3). Infection is more common in women (21\%) and in non-Hispanic Black individuals (39\%; ref. 1). Though most commonly self-limited in the immunocompetent host, infections are lifelong, and in many patients, periodic recurrences are a substantial source of morbidity, with an additional psychosocial burden given stigmatization of genital disease. Moreover, HSV-2 infection is a cofactor increasing the risk of other STIs, including HIV $(3,4)$. In immunocompro- mised populations, more-severe systemic presentations may occur, including liver, lung, and CNS disease. HSV infection in the neonatal period can be devastating, with high mortality and neurologic sequelae $(3,4)$.

Despite substantial vaccine efforts extending into decades, no vaccine approach has proven effective in preventing HSV-2 in randomized human trials (5). Most recently, an HSV-2 glycoprotein D-based recombinant protein vaccine showed initial promise in preventing genital HSV-2 infection (6), though it failed to demonstrate protection in a broader study (7). Notably, potent neutralizing antibodies developed in vaccinated patients yet failed to effectively prevent HSV-2 infection. There are likely two reasons for this

\section{Related Article: https://doi.org/10.1172/JCl142088}

Conflict of interest: Al received research support from D\&D Pharmatech Inc. and Condair, and serves as a scientific advisor to $4 \mathrm{BIO}$. Al is a coinventor on the following pending or issued patents: "A T cell-based immunotherapy for central nervous system viral infections and tumors" (15/596,048); "Manipulation of meningeal lymphatic vasculature for brain and CNS tumor therapy" (PCT/US2019/61624); "Interferon production using short RNA duplexes" (10947543); and "Combination treatments using RIG-I agonists" (17/251,92)

Copyright: (c) 2021, American Society for Clinical Investigation.

Reference information: / Clin Invest. 2021;131(9):e148300. https://doi.org/10.1172/JCl148300.

failure of circulating neutralizing antibodies to confer protection at the mucosa. First, circulating antibodies have limited capacity to cross the intact vaginal epithelial barrier to block viral invasion (8); and second, these antibodies are circumvented by the viral glycoproteins $\mathrm{gE}$, which serves as a decoy $\mathrm{Fc}$ receptor, and glycoprotein $\mathrm{C}$ (gC), which abrogates complement activation (9). However, our understanding of humoral immunity in the context of human HSV-2 prevention and disease is incomplete; additional studies of the $\mathrm{B}$ cell lineage, antibodies, and their role in controlling HSV-2 infection are critical for informing future vaccine design.

\section{B cells and antibody responses to genital herpes}

The role of humoral immunity in HSV-2 in humans is unclear; however, preclinical models suggest that B cells and antibody responses do aid in the immune response to HSV-2 genital disease to some degree. The antigen presentation function of B cells as well as natural antibodies have a limited protective role in the primary infection response to HSV-2 $(10,11)$. HSV-2-immune, B cell-deficient mice only have mildly elevated vaginal viral load but are protected from disease and death after HSV-2 challenge (11, 12). In addition, systemically introduced HSV-2-specific antibodies are unable to prevent HSV-2 infection (12), likely due to the limited access of antibodies across the vaginal epithelial barrier (8, $13,14)$. This raises the question of whether circulating antibodies alone would have any role in preventing HSV-2 infection or facilitating recurrent lesion resolution.

To answer this question, we must consider the role of $\mathrm{T}$ cell-mediated immunity in cutaneous and genital HSV-2 infections $(4,15,16)$. Existing studies in mouse models of genital herpes infection support a crucial role for tissue-resident local $\mathrm{T}$ cells in conferring protection against genital HSV-2 challenge (17-19). Circulating 
Recurrent HSV-2 lesion

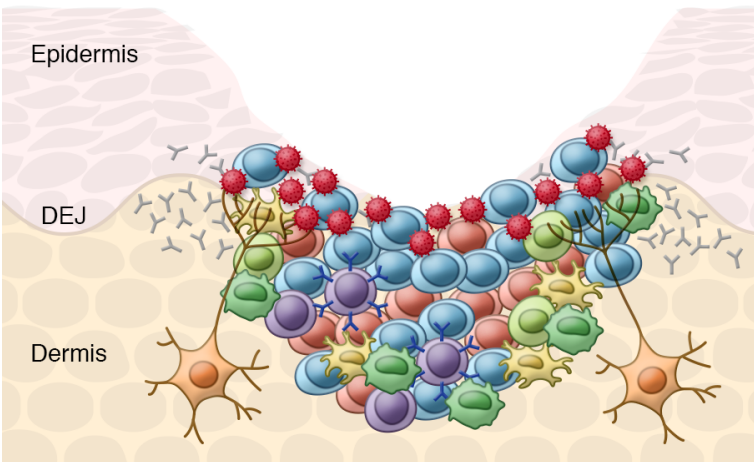

Healing

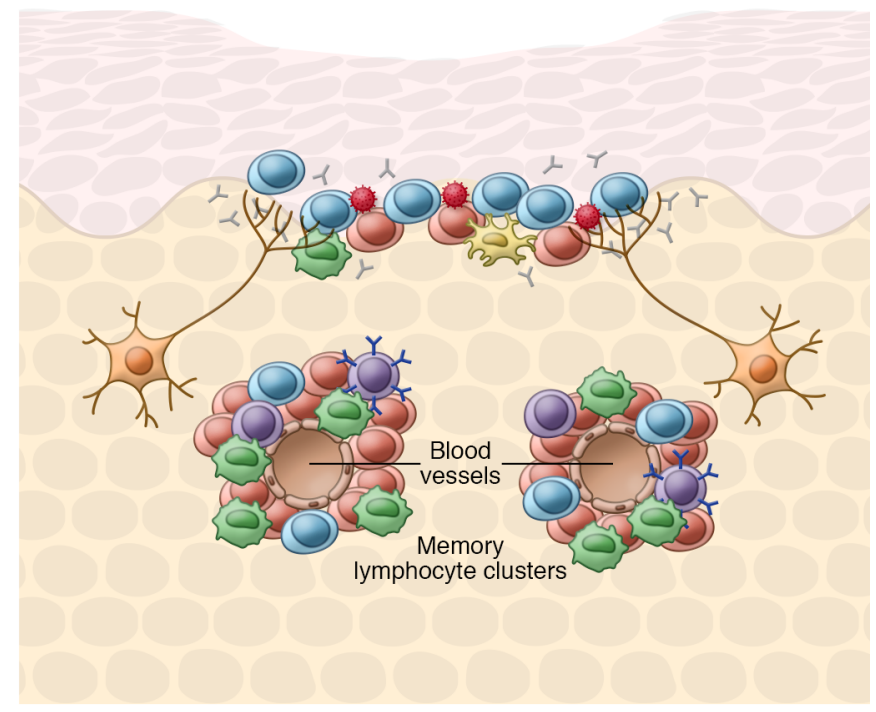

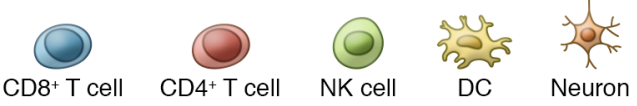
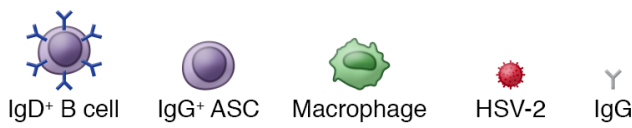

Figure 1. Model of cutaneous and mucosal immune responses to HSV-2 reactivation. In recurrent infections, clusters of antiviral CD4 ${ }^{+}$and $C D 8^{+} \mathrm{T}_{\text {cells }}$ form in the dermis and submucosa, along with dendritic cells and macrophages. Interferon production from T cells helps resolve the viral insult. Naive $B$ cells and ASCs are recruited to these dermal clusters. The latter secrete antibodies that may contribute to blockade of viral spread. During healing, MLCs form. As lesions heal over time, $T$ cell density decreases (though $\mathrm{T}_{\mathrm{RM}} \mathrm{s}$ remain), and B cell populations are no longer, or rarely, observed. DEJ, dermal-epidermal junction.

memory $\mathrm{T}$ cells enter and reside in many organs but fail to establish residency in certain anatomic locations, including the skin and vagina, in the absence of local stimuli (15). Both $\mathrm{CD}^{+}$and $\mathrm{CD} 4^{+}$tissue-resident memory $\mathrm{T}$ cells $\left(\mathrm{T}_{\mathrm{RM}} \mathrm{s}\right)$ have important functional roles in the local antiviral response. $\mathrm{CD}^{+} \mathrm{T}_{\mathrm{RM}} \mathrm{S}$ secrete cytokines and provide superior effector responses $(17,19,20)$, whereas $C D 4^{+} \mathrm{T}_{R M} \mathrm{~s}$ secrete tonic IFN- $\gamma$ and establish local memory lymphocyte clusters (MLCs) with macrophages, which are required for protection following a secondary HSV-2 challenge (18). The importance of tissue-resident T cells in human genital HSV-2 has been shown in multiple studies (21-25). HSV-2 lesion resolution correlates with a strong lymphocyte-derived effector response $(22,24)$. Persistent, localized, HSV-2-specific $\mathrm{CD}^{+} \mathrm{T}$ lymphocytes are more than $90 \% \mathrm{CD}^{+}$in the genital tract of female HSV-2-infected patients (23). Clusters of predominantly $\mathrm{CD} 4^{+} \mathrm{T}$ cells typically occupy perivascular locations in the upper dermis and HSV-2 lesional skin (4), resembling MLCs seen in mouse models of genital HSV-2 infection (18). These MLCs have been characterized primarily for $\mathrm{T}$ cells and their effector functions, but also appear to act as an outpost for recruiting circulating virus-specific B cells during secondary infection.

In a mouse model of genital herpes, intravaginally immunized mice recruited circulating memory B cells and secreted antibodies into the vaginal mucosa after secondary challenge. Conversely, mice that received subcutaneous HSV-2 immunization failed to recruit circulating memory B cells and secrete mucosal antibodies (8). This local antibody-secreting cell (ASC) recruitment to the mucosa was dependent on the CXCR3 chemokines induced by local $\mathrm{CD} 4^{+} \mathrm{T}_{\mathrm{RM}}$ s. Notably, no tissue-resident memory B cells or plasma cells were found 5 weeks after primary genital infection with HSV-2. In contrast, intravenous immunization of macaques with simian immunodeficiency virus pro- moted enrichment of local plasma cells and antibody secretion in the female reproductive mucosal epithelium, which correlated with protection from disease (26). In this issue of the JCI, Ford et al. examined what happens in genital herpes lesions in humans (27).

\section{B cell recruitment to recurrent herpes lesions in humans}

Ford et al. examined longitudinal biopsy specimens of HSV-2-infected skin in 16 participants (14 women and 2 men) with a median age of 50 years (27). Biopsies were performed on clinically active HSV-2 lesions; and healed lesions at 10-14 days after active infection and at 2, 4, and 8 weeks thereafter; and on nonlesional skin as controls. B cells, identified by CD2O and $\mathrm{CD79b}$, were seen in at least two biopsy specimens from each study participant; they were generally more common during active lesions and early healing, yet rarely seen in control biopsies (noted in 3 of 16). Two major populations of $\mathrm{B}$ cells were iden- 
tified: naive $\operatorname{IgD}^{+} \mathrm{B}$ cells and $\operatorname{IgG}^{+}$ASCs. The highest naive $\operatorname{IgD}^{+} \mathrm{B}$ cell and $\operatorname{IgG}^{+}$ ASC densities were observed at the lesional and healing time points (within the first two weeks), which coincided with the time points of maximal $\mathrm{T}$ cell density. These B cell populations were characteristically identified within clusters with T cells, suggesting a possible shared mechanism for recruitment and cooperation. The authors also measured tissue HSV-2-specific antibodies and detected increases compared with antibodies against control antigens, though the time points of maximal density varied across patients and across specimens taken at time of lesion, time of healing, and two to eight weeks after healing. The highest IgG antibody density within lesions was coincident with abundant HSV-2 antigen, suggesting an active role of tissue antibody in the local HSV-2 host response. These studies are the first to our knowledge to identify, characterize, and functionally implicate B cells within the local human recurrent HSV-2 lesions. Given that serum HSV-2 antibody responses remained stable over time, the work of Ford et al. and others highlights the importance of studying humoral host immunity in the mucosal and cutaneous tissues (27).

\section{Implications and future directions}

Through the use of their unique biospecimens and well-executed microscopy-based experiments, Ford and colleagues provide fresh insights into the $\mathrm{B}$ cell lineage and cutaneous HSV-2 infection (27). The identification of antigen-inexperienced B cells, as well as ASCs, suggests a more complex role for $\mathrm{B}$ cells in the response to local HSV-2 reactivation. Though naive B cells are not expected to play a substantial antigen-presenting role (28), their localization within $\mathrm{T}$ cell clusters suggests that the naive B cells may be a source of factors that influence the local inflammatory response to virus. Based on preclinical studies, the enrichment of tissue-localized ASCs portends increased mucosal antibody secretion and protection $(8,26)$. Though tissuelocalized ASCs are rare, identifying these cells provides another clue to strategies that may boost immunity to HSV and viral infections, including enhancing ASC recruitment to infection sites to increase local antibody production.
Importantly, though, questions remain about HSV-2 skin and mucosal immunity. What is the function of naive $\mathrm{B}$ cells in the lymphocyte clusters in recurrent lesions? How are these B cells recruited? Do antiviral antibodies produced by the local ASC result in better viral control? Does antigen presentation by virus-specific B cells promote stimulation of $\mathrm{CD}^{+} \mathrm{T}$ cells, which are the major effector cells that control HSV-2 infection (23)? By virtue of their sample population, the patients included in this study were those who failed to mount an effective immune response to prevent HSV-2 reactivation. While we do have a clearer picture of the immune milieu in human HSV-2 lesions over time, these data do not necessarily inform us of presumptively more-effective immune responses that may prevent infection; though, obviously, such data would be far more difficult to acquire in humans, given these patients would not manifest clinically overt disease.

Incorporating these data with existing studies of human $(4,21-25)$ and preclinical HSV-2 models $(8,10,18)$, we can better construct a hypothesized model of local skin- and mucosa-directed HSV immunity (Figure 1). Both $\mathrm{CD}^{+}$and $\mathrm{CD}^{+} \mathrm{T}_{\mathrm{RM}} \mathrm{S}$ have essential roles in the coordination of local responses, with $\mathrm{CD}^{+} \mathrm{T}_{\mathrm{RM}}$ s providing strong cytotoxic responses and $\mathrm{CD} 4^{+} \mathrm{T}_{\mathrm{RM}} \mathrm{s}$ establishing MLCs that provide a cytokine-rich dermal niche critical for recruiting other cells supporting a local immune response. These additional cell types include CCL5-secreting macrophages as well as B cell populations and ASCs, which secrete antibodies locally that aid in clearing pathogens. With a more comprehensive view of this picture as well as the immunization strategies that maximally promote these localized responses, we will be one step closer to developing a vaccine and therapy that provide protection from HSV infection and/or reactivation.

\section{Acknowledgments}

We thank all the previous and current laboratory members for their work that led to the insights used in this Commentary. Our work in this area is supported by NIH funding (R01AI127429).

Address correspondence to: Akiko Iwasaki, Yale University School of Medicine, 300 Cedar Street, TAC S655B, New Haven, Con- necticut 06520, USA. Phone: 203.785.2919; Email: akiko.iwasaki@yale.edu.

1. Fu X, et al. Seroprevalence of herpes simplex virus type 2 among persons aged 14-49 years - United States - 2005-2008. MMWR. 2010;59(15):456-459.

2. Bradley H, et al. Seroprevalence of herpes simplex virus types 1 and 2 - United States, 1999-2010. J Infect Dis. 2014;209(3):325-333.

3. Gupta R, et al. Genital herpes. Lancet. 2007;370(9605):2127-2137.

4. Schiffer JT, Corey L. Rapid host immune response and viral dynamics in herpes simplex virus-2 infection. Nat Med. 2013;19(3):280-290.

5. Awasthi S, Friedman HM. Status of prophylactic and therapeutic genital herpes vaccines. Curr Opin Virol. 2014;6:6-12.

6. Stanberry LR, et al. Glycoprotein-D-adjuvant vaccine to prevent genital herpes. $N$ Engl J Med. 2002;347(21):1652-1661.

7. Belshe RB, et al. Efficacy results of a trial of a herpes simplex vaccine. $N$ Engl J Med. 2012;366(1):34-43.

8. Oh JE, et al. Migrant memory B cells secrete luminal antibody in the vagina. Nature. 2019;571(7763):122-126.

9. Lubinski JM, et al. Herpes simplex virus type 1 evades the effects of antibody and complement in vivo. J Virol. 2002;76(18):9232-9241.

10. Iijima N, et al. Dendritic cells and B cells maximize mucosal Th1 memory response to herpes simplex virus. J Exp Med. 2008;205(13):3041-3052.

11. Harandi AM, et al. Differential roles of B cells and IFN-gamma-secreting CD4(+) T cells in innate and adaptive immune control of genital herpes simplex virus type 2 infection in mice. J Gen Virol. 2001;82(pt 4):845-853.

12. Dudley KL, et al. Immune protection against HSV-2 in B-cell-deficient mice. Virology. 2000;270(2):454-463.

13. Sherwood JK, et al. Controlled release of antibodies for long-term topical passive immunoprotection of female mice against genital herpes. Nat Biotechnol. 1996;14(4):468-471.

14. Whaley KJ, et al. Passive immunization of the vagina protects mice against vaginal transmission of genital herpes infections. JInfect Dis. 1994;169(3):647-649.

15. Iwasaki A. Exploiting mucosal immunity for antiviral vaccines. Annu Rev Immunol. 2016;34:575-608.

16. Shin H, Iwasaki A. Tissue-resident memory T cells. Immunol Rev. 2013;255(1):165-181.

17. Gebhardt T, et al. Memory T cells in nonlymphoid tissue that provide enhanced local immunity during infection with herpes simplex virus. Nat Immunol. 2009;10(5):524-530.

18. Iijima N, Iwasaki A. T cell memory. A local macrophage chemokine network sustains protective tissue-resident memory CD4 T cells. Science. 2014;346(6205):93-98.

19. Mackay LK, et al. Long-lived epithelial immunity by tissue-resident memory T (TRM) cells in the absence of persisting local antigen presentation. Proc Natl Acad Sci U S A. 2012;109(18):7037-7042.

20. Jiang $X$, et al. Skin infection generates non-migratory memory CD8+ T(RM) cells 
providing global skin immunity. Nature. 2012;483(7388):227-231.

21. Koelle DM, et al. Direct recovery of herpes simplex virus (HSV)-specific T lymphocyte clones from recurrent genital HSV-2 lesions. J Infect Dis. 1994;169(5):956-961.

22. Koelle DM, et al. Clearance of HSV-2 from recurrent genital lesions correlates with infiltration of HSV-specific cytotoxic T lymphocytes. J Clin Invest. 1998;101(7):1500-1508.

23. Posavad CM, et al. Persistence of mucosal
T-cell responses to herpes simplex virus type 2 in the female genital tract. Mucosal Immunol. 2015;8(1):115-126.

24. Schiffer JT, et al. Mucosal host immune response predicts the severity and duration of herpes simplex virus-2 genital tract shedding episodes. Proc Natl Acad Sci U S A. 2010;107(44):18973-18978.

25. Zhu J, et al. Immune surveillance by CD $8 \alpha \alpha+$ skin-resident $\mathrm{T}$ cells in human herpes virus infection. Nature. 2013;497(7450):494-497.
26. Li Q, et al. Live simian immunodeficiency virus vaccine correlate of protection: local antibody production and concentration on the path of virus entry. JImmunol. 2014;193(6):3113-3125.

27. Ford ES, et al. B cells, antibody-secreting cells, and virus-specific antibodies respond to herpes simplex virus-2 reactivation in skin. J Clin Invest. 2021;131(9):e142088.

28. Reichardt $\mathrm{P}$, et al. Naive $\mathrm{B}$ cells generate regulatory $\mathrm{T}$ cells in the presence of a mature immunologic synapse. Blood. 2007;110(5):1519-1529. 